\title{
Ordinal Evaluation: A New Perspective on Country Images
}

\author{
Marko Robnik-Šikonja ${ }^{1}$, Kris Brijs ${ }^{2}$, and Koen Vanhoof ${ }^{2}$ \\ 1 University of Ljubljana, \\ Faculty of Computer and Information Science, \\ Tržaška 25, 1001 Ljubljana, Slovenia \\ Marko.Robnik@fri.uni-lj.si \\ 2 University of Hasselt, Department of Economics, \\ Universitaire Campus, 3590 Diepenbeek, Belgium \\ \{kris.brijs, koen.vanhoof $\}$ @uhasselt.be
}

\begin{abstract}
We present a novel use of ordinal evaluation (OrdEval) algorithm as a promising technique to study various marketing phenomena. OrdEval algorithm has originated in data mining and is a general tool to analyze data with ordinal attributes, including surveys. Its many favorable features, including context sensitivity, ability to exploit meaning of ordered features and ordered response, and robustness to noise and missing values in the data, offer marketing practitioners a perspective, not available with classical analytical toolbox.

We present a case study applying OrdEval algorithm on country-of-origin (COO) information. We demonstrate some interesting advantages it has to offer and show how to extract and interpret new insights allowing marketing practitioners to further optimize the management of products abroad.

Data for the empirical study was gathered by means of 1225 questionnaires. Results indicate that, contrary to the classical view on COO-effects, the processing of country-related cognitions, affects and conations is a non-linear and asymmetric phenomenon. The practical implications of this finding for marketers are discussed more in detail.
\end{abstract}

\section{Introduction}

In recent years we can observe large changes in economy in general and marketing in particular as a result of internet expansion, globalization, and ubiquitous information availability. One of the scientific fields which gained momentum as a result of this was data analysis under various names: statistics, data mining, machine learning, intelligent data analysis, knowledge discovery. Many new data analysis techniques emerged which exploit availability of more and different data from several sources, and increased computational power of nowadays computers. Some examples of these techniques are support vector machines, text analytics, association rules, ensemble techniques, subgroup discovery, etc. These techniques have been accepted into analytics' standard toolbox in many disciplines: genetics, engineering, medicine, finance, vision, statistics, marketing, etc.

The OrdEval algorithm [11] is a novel analytical tool which emerged in data mining context with purpose to evaluate the importance and impact of various factors in the 
given data (e.g., survey). For example, in the analysis of customer satisfaction data for a particular product/service, OrdEval can determine the importance of each product's feature to the overall customer's satisfaction, and also indicate the thresholds where satisfaction with individual feature starts having strong positive or negative impact on the overall satisfaction. The output of OrdEval are probabilistic factors indicating the probability that increase/decrease in the individual feature or the feature's value will have impact on the dependent variable. The intuition behind this approach is to approximate the inner workings of the decision process taking place in each individual respondent, which forms relationship between the features and the response. If such brain introspection would be possible one could observe a causal effect the change of a feature's value has on the response value. By measuring such an effect we could reason about the importance of the feature's values and the type of the attribute. Also, we could determine which values are thresholds for change of behavior. While this is impossible, OrdEval algorithm uses the data sample and approximates this reasoning. For each respondent it selects its most similar respondents and does inference based on them. For example, to evaluate the effect an increase in certain feature value would have on overall satisfaction, the algorithm computes the probability for such an effect from the similar respondents with increased value of that feature. To get statistically valid and practically interesting results the overall process is repeated for large enough number of respondents, and weighted with large enough number of similar respondents. The motivation and contribution of this paper is to demonstrate how OrdEval works in a marketing context, how its output can be visualized and adapted to include information relevant for marketing practitioners, and the new insights into the country-of-origin (COO) problem, which we used as our case study.

We continue with a brief overview of the literature on COO-effects. Without going into the details, it situates the broader (marketing) context in which the technical contributions of this paper are to be seen. Although the accent of this work is on the use and adaptations of OrdEval algorithm in marketing, some background information on what COO-research is all about is necessary for understanding concepts and ideas contained by the present paper. Besides that, we motivate a short excursion into the COO-field by signaling that the contribution of this paper not only lays in technical aspects but also in developing superior consumer information with managerial relevance.

\section{Country-of-Origin Research}

Research on COO-effects is mainly concerned with the effect information about a product's source country exerts on consumer's attitude towards the product. Empirical data overwhelmingly indicates that people indeed are guided somehow by the COO-cue when they are confronted with products coming from abroad. For instance, cars or washing machines 'Made in' Germany are usually preferred over Korean- or Chinesemade models. Below, we briefly discuss how both country image (CI) and product attitude are to be understood.

A product's $\mathrm{COO}$ is often explicitly mentioned in order to favorably influence people's reaction towards the product. Communicating a product's $\mathrm{COO}$ can be done in several manners like for instance by means of a 'Made in' label, a foreign sounding 
brand name or visual imagery. Confronted with these COO-stimuli, an internally stored schema is automatically activated in the consumer's mind. Generally, these memory networks are referred to as country images.

The literature reveals 9 environmental conditions as core aspects of the country image construct. These are cultural identity, political climate, language, national history, natural landscape, meteorological climate, level of technological and economic development, religion, and people's character [9].

Most scholars working within the COO-field operationalize country images as threecomponent attitude constructs. That is, most existing scales for measuring country image capture respondents' beliefs, feelings and intentions towards a country's environmental conditions. These three components which we use throughout this paper as well are normally labeled respectively as the country image's cognitive (mental), affective (emotional) and conative (intentional) dimensions.

Common to each of these three types of COO-effects is that they are theoretically explained as processes where the attitude towards a product is (un)favorably 'biased' in function of how consumers are thinking, feeling or (morally) oriented towards the product's COO. Technically, this biasing effect is believed to be a symmetric and linear mechanism where the scores for product-related beliefs, affects and purchase intentions increase or decrease proportionately to variations in the scores obtained for the country image's constituent components. These processes are better known as upward and downward reinforcement.

However, this traditional view on the functioning of marketing phenomena is challenged by some authors $[1,6,7,4]$. In particular, Prospect Theory and its assumptions of loss aversion and diminishing sensitivity has brought several marketing scholars to alter their views on classic decision making mechanisms such as upward and downward reinforcement. For instance, within the field of customer satisfaction, a number of studies were capable of demonstrating that the relationship between attribute levels and overall satisfaction is rather asymmetric and S-shaped [7, 8, 11]. In contrast with research on customer satisfaction, the COO-literature about asymmetric and non-linear mechanisms is rather scarce. To the best of our knowledge, there are only two trying to explain the asymmetric nature of COO-effects $[12,5]$. As such, this topic is still widely open for further exploration.

In light of these ideas, we intend to reconsider the classical explanations for the technical mechanisms in support of COO-effects. In our attempt to address this issue, we first demonstrate a technique that is capable of capturing the type of processes we would like to investigate.

\section{OrdEval}

Only recently, Robnik-Šikonja and Vanhoof [11] introduced the Ordered Evaluation (OrdEval) algorithm. It can be used for analysis of any data where the dependent variable has ordered values, meaning that is suitable also for surveys where answers are given in the graded manner. The methodology uses conditional probabilities called 'reinforcement factors' as they approximate upward and downward reinforcement of the feature value. For each value of the feature we obtain estimates of two conditional 
probabilities: the probability that the response value increases given the increase of the feature value (upward reinforcement), and the probability that response value decreases given the decrease of the feature value (downward reinforcement). To take the context of other features into account, these probabilities are computed in the local context, from the most similar instances. The visualization of these factors gives clear clues about the role of each feature, the importance of each value and the threshold values. To understand the idea of OrdEval algorithm, the feature should not be treated as a whole. Rather we shall observe the effect a single value of the feature may have. Assume for a moment that we could observe the inner workings of the decision process which forms relationship between the features and the response. In other words, suppose that we can observe a causal effect the change of a feature's value has on the response value. By measuring such an effect we could reason about the importance of the feature's values. Also, we could determine which values are thresholds for change of behavior and we could characterize the behaviors. This is of course impossible, but OrdEval algorithm uses the data sample and approximates this reasoning.

To explain the idea of the approach we need some definitions. Let $R$ be a randomly selected observation and $S$ the observation most similar to it. Let $j$ be the value of the feature $A_{i}$ at observation $R$. We observe the necessary changes of response value and features ( $A_{i}$ with value $j$ in particular) which would change $S$ to $R$. If these changes are positive (increase of response and/or feature values), let us define the following probabilities.

- $P\left(C_{i, j}^{p}\right)$ is a probability that the response value of $R$ is larger than the response value of its most similar observation $S . P\left(C_{i, j}^{p}\right)$ is therefore the probability that the positive change in similar observation's response value is needed to get from $S$ to $R$ (note that for $R$ the value of attribute $A_{i}$ is $j$ ).

- $P\left(A_{i, j}^{p}\right)$ is a probability that $j$ (the value of feature $A_{i}$ at $R$ ) is larger than the value of feature $A_{i}$ at its most similar observation $S$. By estimating $P\left(A_{i, j}^{p}\right)$ we gather evidence of the probability that the similar observation $S$ has lower value of $A_{i}$ and the change of $S$ to $R$ is positive.

- $P\left(C^{p} A_{i, j}^{p}\right)$ is a probability that both the response and $j$ (the value of feature $A_{i}$ at $R$ ) are larger than the response and feature value of its most similar observation $S$. With $P\left(C^{p} A_{i, j}^{p}\right)$ we estimate the probability that positive change in both the response and $A_{i}$ value of similar instance $S$ is needed to get the values of $R$.

Similarly, for negative changes which would turn $S$ into $R$ (decrease of response and/or feature values), we define $P\left(C_{i, j}^{n}\right), P\left(A_{i, j}^{n}\right)$, and $P\left(C^{n} A_{i, j}^{n}\right)$. The output of the algorithm are two factors, upward and downward reinforcement, computed for each value of each feature. These factors measure the upward/downward trends exhibited in the data. The upward reinforcement of the $i$-th feature's value $j$ is defined as

$$
U_{i, j}=P\left(C_{i, j}^{p} \mid A_{i, j}^{p}\right)=\frac{P\left(C^{p} A_{i, j}^{p}\right)}{P\left(A_{i, j}^{p}\right)}
$$

This factor reports the probability that a positive response change is caused by the positive feature change. This intuitively corresponds to the effect the positive change in 
feature's value has on the response. Similarly the downward reinforcement is defined as

$$
D_{i, j}=P\left(C_{i, j}^{n} \mid A_{i, j}^{n}\right)=\frac{P\left(C^{n} A_{i, j}^{n}\right)}{P\left(A_{i, j}^{n}\right)}
$$

and reports the effect the decrease of attribute's value has on the decrease of the class' value. The $U_{i, j}$ and $D_{i, j}$ factors are efficiently estimated by the OrdEval algorithm which we present in Fig. 1 in a simplified form intended for easier comprehension.

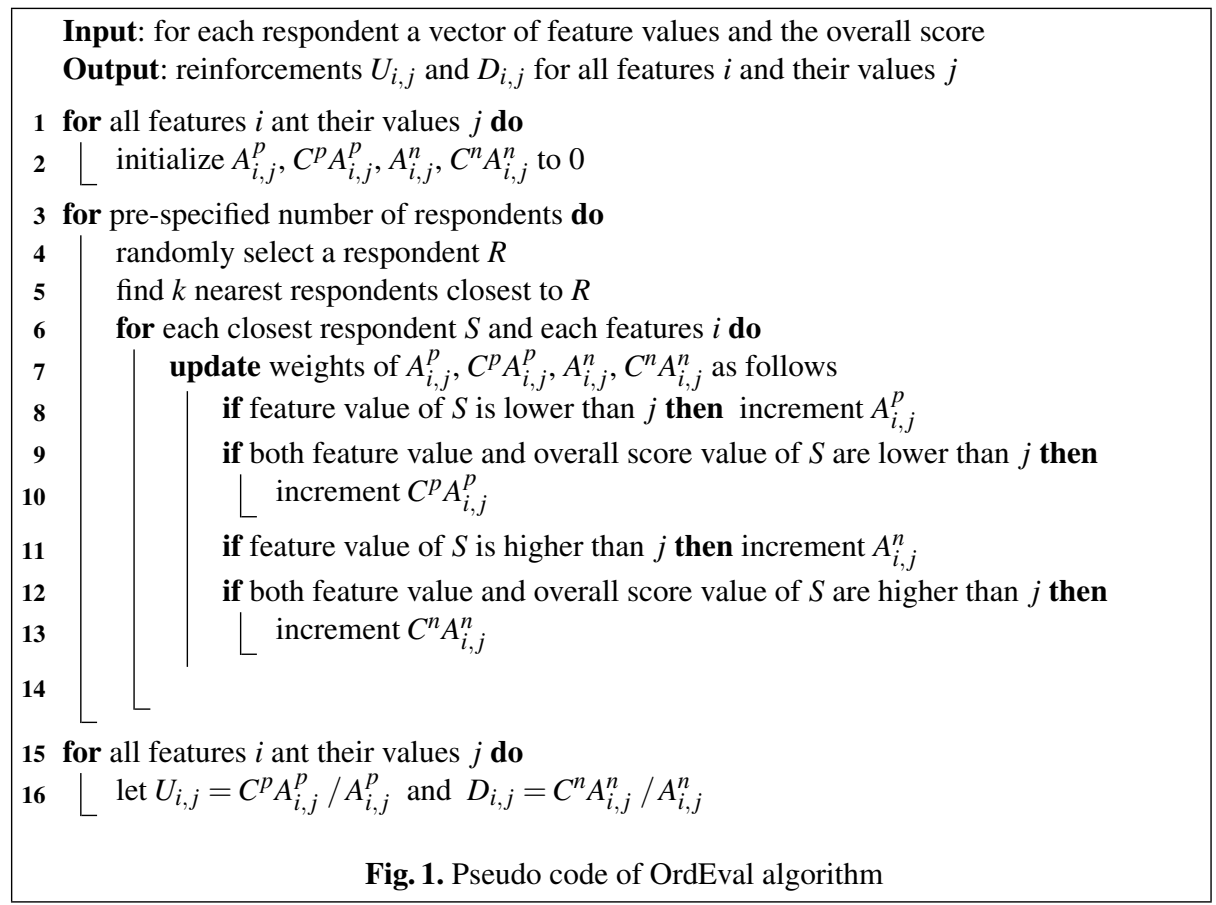

The algorithm assumes that the cause of the differences in overall score are the differences in the attributes' values and gives these values some credit for that, but only if the sign of the differences in class and attribute is the same. It first sets counters of (co)occurring changes to zero (lines 1 and 2). Than it randomly selects a respondent $R$ (line 4) and searches for its $\mathrm{k}$ nearest respondents (line 5). For each of these most similar respondents it updates the counters for all the features depending on the overall scores and feature values of the randomly selected respondent and the near respondents (lines 7 - 14): if the feature value of the near instance is lower than the value of the random instance (line 8) then the change is positive and we update $A_{i, j}^{p}$ for the value $j$ of the given feature $i$ ( $j$ is the value of feature $i$ for respondent $R$ ). If additionally the overall score of the similar respondent is lower than the score of the random respondent (line 9) then the change in both overall score and feature is positive and we update 
$C^{p} A_{i, j}^{p}$ for given feature $i$ and its value of random respondent $j$ (line 10). Similarly we do for negative changes in feature and overall score (lines 11-13). We repeat the whole process (lines 3 - 14) for a pre-specified number of iterations. Conservatively we can set this number to be equal to the number of respondents, but we get useful results even if we run only a few iterations (e.g., logarithm of the number of respondents). Finally the upward and downward enforcement factors for all the values of attributes are computed as conditional probabilities (lines 15-16).

To show the behavior and usability of the algorithm we first define a simple artificial problem which is motivated by the Behavioral Decision Theory, stating that there are several distinct manners according to which marketing stimuli (like the COO) can be used during the formation of product attitude [4].

Our data set is described by six important and two irrelevant features. The important features correspond to different feature types from the marketing theory: two basic features $\left(B_{\text {weak }}\right.$ and $\left.B_{\text {strong }}\right)$, two performance features $\left(P_{\text {weak }}\right.$ and $\left.P_{\text {strong }}\right)$, two excitement features $\left(E_{\text {weak }}\right.$ and $\left.E_{\text {strong }}\right)$, and two irrelevant features $\left(I_{\text {uniform }}\right.$ and $\left.I_{\text {normal }}\right)$. The values of all features are randomly generated integer values from 1 to 5 , indicating for example score assigned to each of the features by the survey's respondent. The dependent variable for each instance (class) is the sum of its features' effects, which we scale to the uniform distribution of integers 1-5, indicating, for example, an overall score assigned by the respondent.

$$
C=b_{w}\left(B_{\text {weak }}\right)+b_{s}\left(B_{\text {strong }}\right)+p_{w}\left(P_{\text {weak }}\right)+p_{s}\left(P_{\text {strong }}\right)+e_{w}\left(E_{\text {weak }}\right)+e_{s}\left(E_{\text {strong }}\right)
$$

The effects of attributes are as follows.

- Basic features are taken for granted by customers; high score in these features does not significantly increase the overall score, while a low score has a decreasing effect on dependent variable. We define two variants of basic features, one with weaker and another with stronger negative impact:

$$
b_{w}(A)=\left\{\begin{array}{r}
-2 ; A<=2 \\
0 ; A>=3
\end{array}\right\}, \quad b_{s}(A)=\left\{\begin{array}{l}
-4 ; A<=3 \\
-2 ; A=4 \\
-0 ; A=5
\end{array}\right\} .
$$

- Performance features have positive correlation with overall score: the higher the value of the attribute the bigger the effect on the overall score. We define the performance effects as

$$
p_{w}(A)=\left\{\begin{array}{r}
-3 ; A=1 \\
-2 ; A=2 \\
-0 ; A=3 \\
2 ; A=4 \\
3 ; A=5
\end{array}\right\}, \quad p_{s}(A)=\left\{\begin{array}{r}
-5 ; A=1 \\
-3 ; A=2 \\
-0 ; A=3 \\
3 ; A=4 \\
5 ; A=5
\end{array}\right\} .
$$

- Excitement features describe properties of product/service which are normally not very important to the users, but can cause excitement if the score is very high. We 
define two grades of excitement effect as

$$
e_{w}(A)=\left\{\begin{array}{l}
0 ; A<=4 \\
1 ; A=5
\end{array}\right\}, \quad e_{s}(A)=\left\{\begin{array}{l}
0 ; A<=4 \\
4 ; A=5
\end{array}\right\} .
$$

We generated 1000 instances for this data set. While the value distribution and the independence of features are unrealistic, note that we have experimented also with more realistic distributions as well as with different types of correlation, but the results and conclusions remain unchanged. Table 1 shows the upward and downward reinforcement factors the OrdEval algorithm returned for this data set. The direct interpretation and analysis of these numbers is of course possible, but visualization makes it easier.

Table 1. Upward and downward reinforcement factors for the pedagogical data with different types of features.

\begin{tabular}{lrrrrrrrrr}
\hline & $P_{\text {weak }}$ & $P_{\text {strong }} B_{\text {weak }}$ & $B_{\text {strong }}$ & $E_{\text {weak }}$ & $E_{\text {strong }}$ & $I_{\text {uniform }}$ & $I_{\text {normal }}$ \\
\hline$U_{., 1}$ & 0 & 0 & 0 & 0 & 0 & 0 & 0 & 0 \\
$U_{., 2}$ & 0.42 & 0.45 & 0.29 & 0.30 & 0.33 & 0.33 & 0.33 & 0.32 \\
$U_{., 3}$ & 0.50 & 0.69 & 0.51 & 0.26 & 0.30 & 0.29 & 0.29 & 0.30 \\
$U_{., 4}$ & 0.53 & 0.73 & 0.30 & 0.54 & 0.31 & 0.24 & 0.32 & 0.30 \\
$U_{., 5}$ & 0.39 & 0.45 & 0.33 & 0.47 & 0.39 & 0.72 & 0.29 & 0.33 \\
\hline$D_{., 1}$ & 0.38 & 0.44 & 0.26 & 0.28 & 0.31 & 0.32 & 0.300 & .29 \\
$D_{., 2}$ & 0.46 & 0.66 & 0.49 & 0.25 & 0.27 & 0.29 & 0.27 & 0.28 \\
$D_{., 3}$ & 0.51 & 0.69 & 0.28 & 0.52 & 0.29 & 0.24 & 0.28 & 0.28 \\
$D_{., 4}$ & 0.36 & 0.43 & 0.30 & 0.45 & 0.36 & 0.71 & 0.26 & 0.28 \\
$D_{., 5}$ & 0 & 0 & 0 & 0 & 0 & 0 & 0 & 0 \\
\hline & & & & & & & & &
\end{tabular}

The slope visualization proposed by [11] (upward and downward reinforcement are represented with the steepness of the line segment between two consecutive feature values) is unusual for marketing research practitioners and, as we argue below, does not convey all the information necessary for this specific field. We therefore propose a marketing friendly visualization of the OrdEval results on Fig. 2, which contains results for each feature separately.

The 8 graphs are a sort of bar charts with addition of confidence intervals. For each graph a left-hand side with blue bars contains downwards reinforcements for each feature score separately. Upwards reinforcement factors for all the scores are represented with red bars on the right-hand side of each graph. Before we explain the results let us give a motivation for grey box-and-whiskers graphs on top of each reinforcement bar.

There are two problems with reinforcement factors when used in marketing:

- Imbalanced value distribution: it is quite common that for certain features some scores are almost non-existent (e.g., extremely low score of a basic feature is very rare - such a customer, would probably change the supplier), and also the reverse might be true, namely on a scale 1-5 it is not uncommon that almost all the scores are 4 and 5. Such imbalance also has consequences for reinforcement factors, since the probability of the increased/decreased overall score might be an artefact of the skewed distribution of values. 


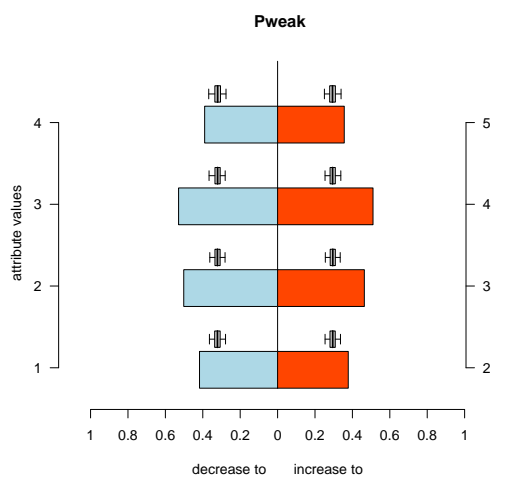

decrease to increase to

Bweak

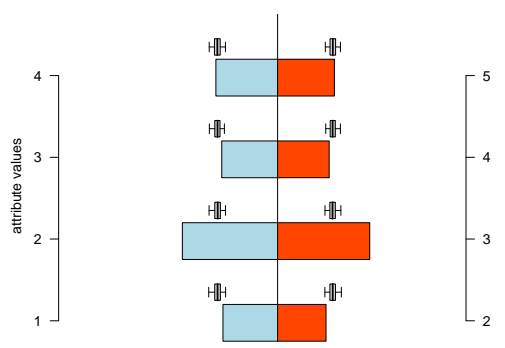

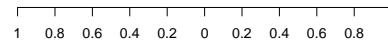

decrease to increase to

Eweak

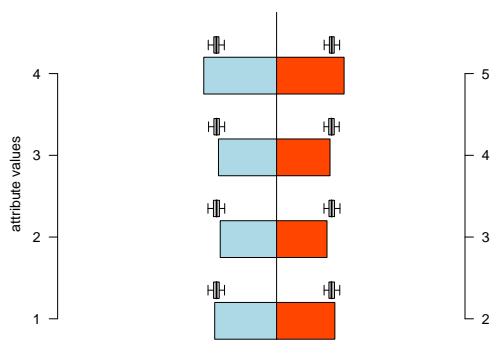

\begin{tabular}{lllllllllll}
\hline 1 & $\mid$ & $\mid$ & $\mid$ & $\mid$ & $\mid$ & $\mid$ & $\mid$ & $\mid$ & $\mid$ \\
1 & 0.8 & 0.6 & 0.4 & 0.2 & 0 & 0.2 & 0.4 & 0.6 & 0.8
\end{tabular}

decrease to increase to

luniform

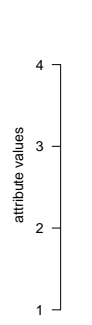
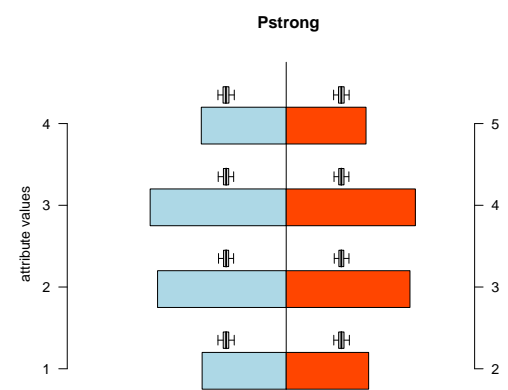

\begin{tabular}{lllllllllllll}
\hline 1 & 0.8 & 0.6 & 0.4 & 0.2 & 0 & 0.2 & 0.4 & 0.6 & 0.8 & 1
\end{tabular}

decrease to increase to

Bstrong

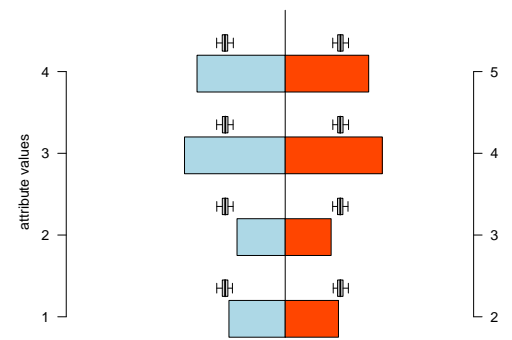

\begin{tabular}{lllllllllllll}
\hline 1 & 0.8 & 0.6 & 0.4 & 0.2 & 0 & 0.2 & 0.4 & 0.6 & 0.8 & 1
\end{tabular}

decrease to increase to

Estrong

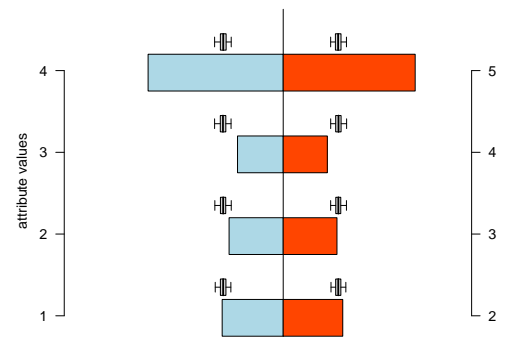

\begin{tabular}{lllllllllllll}
\hline & $\mid$ & $\mid$ & 1 & $\mid$ & 1 & 1 & $\mid$ & $\mid$ & $\mid$ & \\
1 & 0.8 & 0.6 & 0.4 & 0.2 & 0 & 0.2 & 0.4 & 0.6 & 0.8 & 1
\end{tabular}

decrease to increase to

Inormal

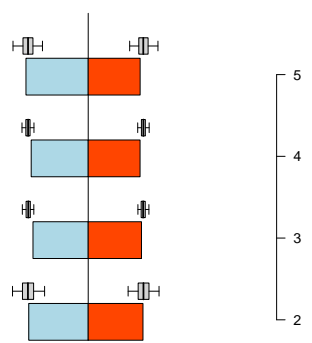

\begin{tabular}{llllllllll}
\hline & & 1 & 1 & & & & & & \\
0.8 & 0.6 & 0.4 & 0.2 & 0 & 0.2 & 0.4 & 0.6 & 0.8 & 1
\end{tabular}

decrease to increase to

Fig. 2. Visualization of reinforcement factors and their confidence intervals on the problem with different types of features. 
- Lack of information about significance of the reinforcement factors: the user does not know what expected range of a certain reinforcement factor is and weather the computed score is significantly different from the uninformative feature.

To solve both problems we compute confidence intervals for each reinforcement factor. Since we cannot assume any parametric distribution and have to take the context of similar respondent into account we proceed as follows:

1. for each feature we construct e.g., $n=200$ features with bootstrap sampled values from the original feature (alternatively the values can be randomly shuffled), we call these features normalizing features,

2. when searching similar respondents we only take original features into account, but we estimate also the reinforcement factors of randomly constructed features,

3. for each reinforcement factor $U_{i, j}$ and $D_{i, j}$ (upward and downward reinforcement for each value of each feature) we perform a statistical testing based on bootstrap estimates [3].

(a) the null hypothesis states that the reinforcement factor is uninformative, i.e., it is equal to the median of its random normalizing features

(b) the alternative hypothesis is one-sided, as we are interested if the reinforcement of the original feature is larger than the random normalizing reinforcement

(c) set fixed confidence level, e.g. $\alpha=0.05$

(d) sort the reinforcement factors of random normalizing features in ascending order

(e) if the reinforcement factor of the original feature is larger than $n(1-\alpha)$ th sorted factor we can reject the null hypothesis, and assume that the computed reinforcement contains significant information

4. the sorted reinforcement factors are the source of information for box-and-whiskers plot: the box is constructed from the 1st and 3rd quartile, middle line is median, while the whiskers are $100 \alpha / 2$ and $100(1-\alpha / 2$ percentiles (e.g. 2.5 and 97.5 percentiles) giving the borders of confidence interval (e.g., $95 \%$ confidence interval).

On Fig. 2 reinforcement factors (blue and red bars) reaching beyond the box-andwhiskers therefore contain significant information. Since the way we construct confidence intervals is not sensitive to the number of instances, these intervals are valid even for low number of scores. We can observe that the algorithm has captured the important landmarks of the features:

- for performance features $P_{\text {weak }}$ and $P_{\text {strong }}$ (two graphs in the top row) all the upward and downward reinforcements are significant, and the relative length of the bars is roughly proportional to the difference between impacts of the values,

- for basic feature $B_{\text {weak }}$ (left-hand graph in the second row) the thresholds at values 2 and 3 (increasing feature from 2 to 3 strongly increases the overall score, and decreasing this feature from 3 to 2 strongly decreases the overall score).

- for basic attribute $B_{\text {strong }}$ (right-hand graph in the second row) the upward thresholds at values 3 and 4 and downward reinforcement thresholds 4 and 5,

- for excitement features $E_{w e a k}$ and $E_{\text {strong }}$ (third row graphs) the jump from 4 to 5 and back is detected, in upward and downward enforcement, respectively. The reinforcements are larger for $E_{\text {strong }}$ as expected. 
- irrelevant random features $I_{\text {uniform }}$ and $I_{\text {normal }}$ have no significant values (bottom row).

Note that only the reinforcement for the thresholds we have defined, are significantly larger than the boundaries of confidence intervals defined by the normalization features.

The properties of the used approach relevant to our study in particular, and in more general terms, to the COO-field at large, are manifold. Firstly, there is substantial context sensitivity. Typically the features are highly conditionally dependent upon the response and have to be evaluated in the context of other features. OrdEval is intrinsically contextual and assumes neither independence nor some fixed distribution of the features. The context of other features is handled through the distance. By using different distance measures and different features in the calculation of the distance, we are even in a position to use different contexts, e.g., we could use some background socio-economic information to calculate the similarity of respondents. Secondly, there is the ability to handle ordered features and ordered response and to use the information the ordering contains. The order of the attribute's values contains information which is comparable but not the same as values of numerical features, e.g., values poor, good, very good, excellent are ordered in expressing certain attitude but this ordering is not necessarily linear. Thirdly, we have awareness of the meaning implied by the ordering of the answers and the positive (negative) correlation of changes between feature values and the response (e.g., if the value of the feature increases from poor to good, we have to be able to detect both positive and negative correlation to the change of the overall response value). Fourthly, OrdEval has the ability to handle each value of the feature separately, e.g., for some features the value of good and very good have identical neutral impact on the response, value poor may have strong negative, and value excellent highly positive impact. We are able to observe and quantify each feature's values separately and thereby identify important thresholds. Next to that, visualization of the output allows experts to use it as a powerful exploratory data analysis tool, e.g., to identify type of features and the impact of their individual values. Also, the output is in the form of probabilities. Probability theory is commonly used and therefore the results in form of probabilities are comprehensible and interpretable by a large audience and can also be used operationally. Finally, we have fast computation and robustness to noise and missing values. A study of the family of the algorithms similar to OrdEval has shown that feature evaluation is possible and reliable even for extremely noisy data [10].

The following section describes the methodological design of an empirical study we conducted to test the OrdEval algorithm and to investigate the true nature of the mechanisms supporting COO-effects.

\section{Case Study: COO}

We demonstrate the analysis possible with OrdEval on a specific marketing-related issue, i.e., the functioning of COO-effects. A complete description of the data set, methodological design of the survey and questionnaire are given in [2]. Here we give only the relevant selection.

The products selected for the study were DVD-players (utilitarian) and beer (hedonic). The countries-of-origin selected were Spain and Denmark. Both countries were 
sufficiently familiar to respondents and mutually different on a number of countryspecific aspects. Data was gathered by means of two surveys (one for Spain/Spanish products and one for Denmark/Danish products). These were distributed to respectively 616 and 609 graduate students of Belgian nationality. Several meta-analyses report that there are no significant differences in the estimates of COO-effects sizes between student and non-student samples [13].

The questionnaire consisted of 7 sections. First, subjects indicated sex and age. For all the remaining questions the subjects evaluated each of the country characteristics on a 7 point scale. Subjects were asked on their cognitions about nine environmental conditions (cultural identity, political climate, language, history, landscape, climate, technological and economic development, religion and people's character). Ten questions measured subjects' feelings towards $\mathrm{COO}$ following by four items retrieving respondents' behavioral intentions towards Spain or Denmark (I would like to shop, work, buy products, do business with and invest in country X). Next reliability, durability, performance and easiness of use were queried for DVD-players and taste, naturalness, aroma and prestige for beer. Subjects also evaluated DVD-players and beer (overall quality, likeability, appeal). Finally, purchase intentions were assessed with three questions (i.e., I would be willing to buy, consider buying, there is a chance I would buy product $\mathrm{X}$ ). The 7 point scale was later turned into the 3 point scale (low, medium, high) for all variables as follows: $1,2 \rightarrow$ low; $3,4,5 \rightarrow$ medium; $6,7 \rightarrow$ high. Additional summarization over relevant variables was performed to get an overall score for the cognitive, emotional, and conative dimension which we used in our study.

\subsection{Results}

For each combination of product (Beer, or DVD-player) and country (Spain or Denmark) we computed reinforcement factors with OrdEval and generated a graph with visualization of reinforcement factors. The most interesting results we report below.

Reinforcement factors for Spanish beer (see Fig. 3) are followed by the results for Spanish DVD-players (Fig. 4). Each of the visual outputs can be read in a similar fashion. The three columns contain graphs for each CI dimension (feelings, conative, and cognitive) serving as an overall score (dependent variable). The three rows contain country-product score (product evaluation, beliefs, and purchase intentions) serving as independent variable. While these graph look like showing one dimensional dependencies, note that this is not so, as the context of all independent variables is taken into account through similarity of instances. Each of these independent variables can have three different values (low, medium and high). Upward/downward reinforcement is indicated with red/blue bars on right/left hand side of each of the nine graphs.

Several things can be learned from these graphs, like (non)linearity, (a)symmetry, threshold values and significance of reinforcements. For linear features one would expect that the stronger the preference for certain COO-dimension the better the country image. For example on Fig. 3 all the features show such tendency for the positive feelings toward Spain (first column). To detect symmetry, comparison of the red and blue bars on the same level allows us to determine what the valence of country image effects is like, namely the visual outputs on the same levels allow comparison between upward and downward reinforcement. In most cases on Fig. 3 there is a clear tendency towards 


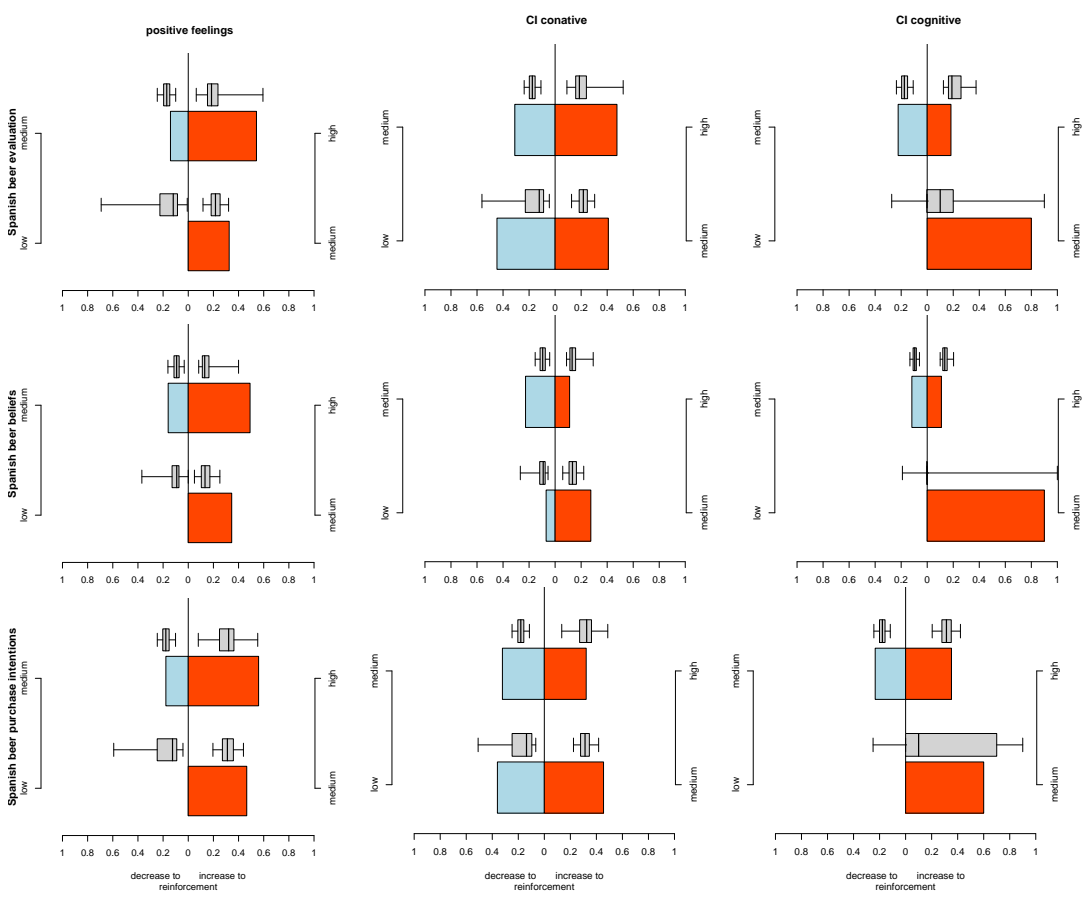

Fig. 3. Visualization of OrdEval results for Spanish beer.

asymmetry. Threshold values are detected by observing for which value the reinforcement factors (red and blue bars) become significant and exceed in length the whiskers of gray box and whiskers graph above it. In the same way we detect significant reinforcements - they exceed whiskers of confidence interval. Since confidence intervals do not assume any distribution and also take number of instances into account we get practically useful information.

Within the Spanish survey, results obtained for beer (Fig. 3) and DVD-players (Fig. 4) are very consistent. Contrary to the traditionally supported linear and symmetric perspective towards COO-effects, application of the OrdEval algorithm indicates that country image (CI) is operating in a non-linear and asymmetric manner. The non-linearity implies that the probability of increase or decrease in the dependent variable (i.e., product attitude) varies in function of what the precise value obtained for the independent variable (i.e., the CI) is like. The asymmetry implies that the probability of increase or decrease in the dependent variable differs between upward and downward reinforcement. If we apply this overall finding to each of CI's three constituent components taken separately, we can come to the following more detailed conclusions.

The finding of non-linearity implies that the CI's cognitive component probably will cause an effect on product attitude only in those specific cases where high scores for the CI's cognitive component change into moderate scores. More in particular, the critical threshold for the ci's cognitive component to exert an effect on product attitude 


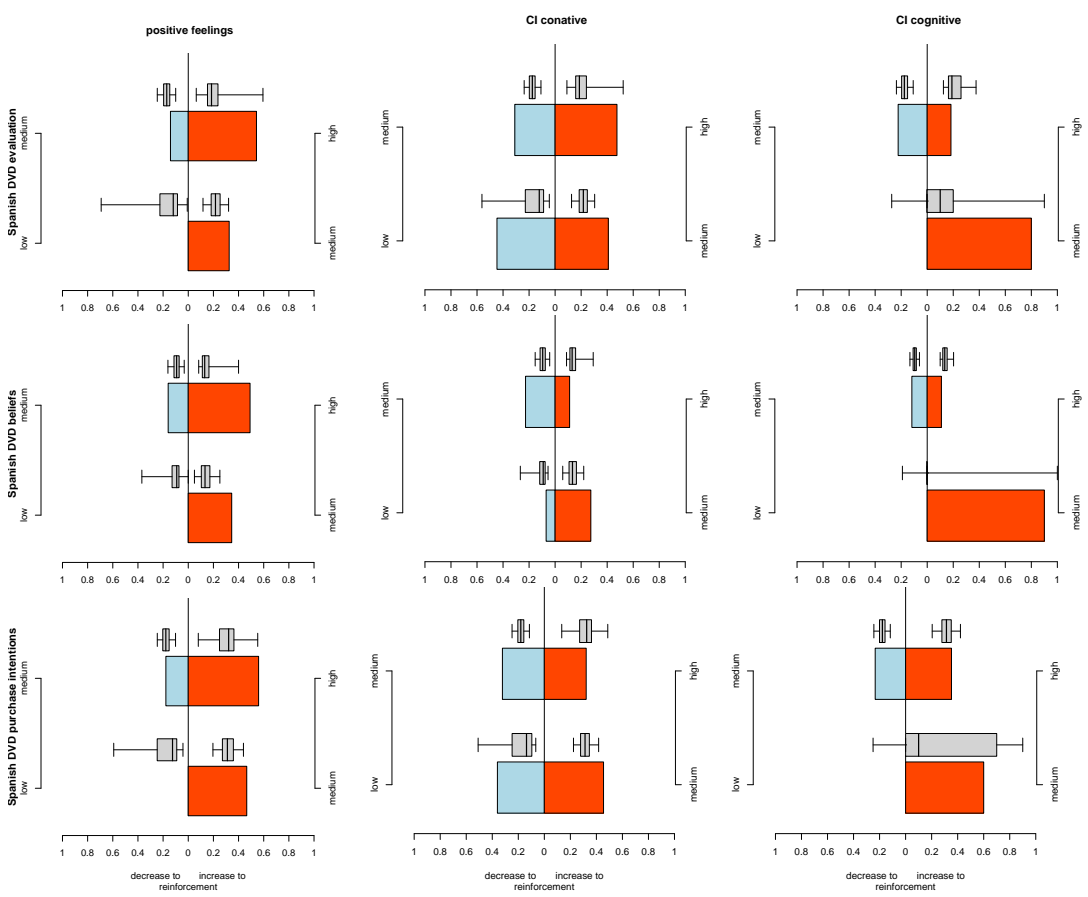

Fig. 4. Visualization of OrdEval results for Spanish DVD-players.

is situated at the transition from high to medium. The finding of asymmetry means that the probability of an effect on product attitude to occur can only be supported for downward reinforcement effects. Put differently, while a decrease in the value for the CI's cognitive component (from high to moderate) can be expected to (negatively) affect product attitude, the opposite scenario, i.e., an eventual increase in the value for the CI's cognitive component will fail to produce a (positive) effect on product attitude.

Non-linearity for the CI's affective component manifests itself somewhat differently compared with the CI's cognitive component in that the probability of CI's affective component to exert an effect on product attitude can be established for both changes from low into moderate and from moderate into high values. However, in general, the probability of an effect to occur is higher for changes from moderate to high values than for changes from low into moderate values. Thus, the critical threshold for the CI's affective component can be situated at the transition from medium to high. In line with the outcome for the CI's cognitive component, the CI's affective component operates asymmetrically in that it is expected to influence product attitude almost exclusively in cases where the values for the CI's affective component increase.

Finally, results obtained for the CI's conative component indicate that subjects' product attitude seems to be sensitive to changes in values for the CI's conative component in a different manner compared to how their product attitude reacts to changes in values obtained for the CI's cognitive and affective components. As already discussed, 
changes in values for the CI's cognitive and affective component influence product attitude only in one single direction (i.e., a downward effect for the cognitive component and an upward effect for the affective component). For the CI's conative component, probability scores indicate that both upward and downward effects can be expected to occur. However, comparable to the findings for the two other components of CI, the effects generated by the CI's conative component are supported by a similar mechanism, i.e., non-linear and asymmetric. Both upward and downward reinforcement effects triggered by the CI's conative component have a specific threshold value above or underneath which no further change in product attitude is to be expected. For upward reinforcement, this critical value lies at the transition from a scalar value of low to medium, for downward reinforcement the critical value is to be situated at the transition from high to medium. The asymmetry is reflected in that an increase in value from low to medium causes upward reinforcement without the opposite process of a decrease in value from medium to low resulting in a downward reinforcement effect. In line with this, downward reinforcement for the CI's conative component is generated in case there is a decrease in value from high to medium without upward reinforcement taking place for an increase in value from medium to high.

Results for the Danish survey are very much alike those for the Spanish survey, left aside a few minor differences. Analysis of the data by means of the OrdEval algorithm again shows that the CI's three basic components are operating in a non-linear and asymmetric manner.

\section{Conclusions}

We separate our conclusions into two groups, first COO related relevant for marketers, and the second a methodological one, relevant for data miners.

From a marketing point of view, we can state that this study clearly raises some issues with regard to the traditional view on COO-effects as a linear and symmetric phenomenon. Different from previous publications on COO-effects, we consider COOeffects as a non-linear phenomenon. As a consequence, marketers, in order to deal effectively with a product's $\mathrm{COO}$, should be knowledgeable about the very precise critical or threshold value of CI. Next to that, we see COO-effects as an asymmetric phenomenon. This implies that marketers should be aware of the fact that upward and downward reinforcement effects in product attitude operate in full independence from each other.

From a data mining point of view the paper has adapted a general methodology for analysis of ordered data to the specifics of marketing. OrdEval algorithm possesses several favorable properties like context sensitivity, ability to handle ordered features and ordered response, awareness of the meaning implied by the ordering, ability to handle each value of the feature separately, output is in the form of probabilities, and fast computation and robustness to noise and missing values. In this paper we proposed an algorithm to compute confidence intervals for reinforcement factors thereby solving important problems which prevented its practical use. For example imbalanced value distribution is quite a common phenomenon but it has severe consequences for reinforcement factors, since the probability of the increased/decreased overall score might be an artefact of the skewed distribution of values. Another such obstacle is information 
about significance of the reinforcement factors: the user does not know what expected range of a certain reinforcement factor is and weather computed score is significantly different from the uninformative feature. By computing distribution independent confidence intervals we provide information on reliability of the reinforcement scores which give them practical importance and enables confident decision making. Additionally the proposed visualization of the reinforcement factors enables detection of (non)linearity, (a)symmetry, threshold values and significance of the results.

\section{Bibliography}

[1] E. W. Anderson and M. Sullivan. The antecedents and consequences of customer satisfaction for firms. Science, 12(2):125-143, 1993.

[2] K. Brijs. Unravelling country-of-origin: Semiotics as a theoretical basis for a meaning-centred approach towards country-of-origin effects. $\mathrm{PhD}$ thesis, University of Hasselt, 2006. URL http://hdl.handle.net/1942/1819.

[3] B. Efron and R. J. Tibshirani. An introduction to bootstrap. Chapman \& Hall, New York, 1993.

[4] H. J. Einhorn and R. M. Hogarth. Behavioral decision theory: Processes of judgement and choice. Annual Review of Psychology, 32:53-88, 1981.

[5] D. Maheswaran and C. Y. Chen. Nation equity: Incidental emotions in countryof-origin effects. Journal of Consumer Research, 33(3):370-376, 2006.

[6] K. E. Matzler, F. Bailom, H. Hinterhuber, B. Renzl, and J. Pichler. A reconsideration of the importance-performance analysisthe asymmetric relationship between attribute-level performance and overall customer satisfaction: A reconsideration of the importance-performance analysis. Industrial Marketing Management, 33: 271-277, 2004.

[7] V. Mittal and W. Kamakura. Satisfaction, repurchase intention, and repurchase behavior: Investigating the moderating effect of customer characteristics. Journal of Marketing Research, 38 (February):131-142, 2001.

[8] G. Peeters and J. Czapinski. Positive-negative asymmetry in evaluations: The distinction between affective and informational negativity effect. European Review of Social Psychology, 1:33-60, 1990.

[9] J. Pharr. Synthesizing country-of-origin research from the last decade: is the concept still salient in the era of global brands? Journal of Marketing Practice and Theory, 13(4,):34-45, 2006.

[10] M. Robnik-Šikonja and I. Kononenko. Theoretical and empirical analysis of ReliefF and RReliefF. Machine Learning Journal, 53:23-69, 2003.

[11] M. Robnik-Šikonja and K. Vanhoof. Evaluation of ordinal attributes at value level. Data Mining and Knowledge Discovery, 14:225-243, 2007.

[12] J. W. Story. The effects of perceived technological incongruence on perceptions of fit between countries, products, and attributes. Journal of Business Research, 58(10):1310-1319, October 2005.

[13] P. W. J. Verlegh and J.-B. E. M. Steenkamp. A review and meta-analysis of country-of-origin research. Journal of Economic Psychology, 20(5):521-546, October 1999. 